\title{
Nuevas vías de comunicación científica experimentadas desde una revista
}

New scientific communication channels tested from a journal

\author{
Tomàs BAIGET \\ El Profesional de la Información, EPI SCP, Gran Vía Corts Catalanes 440, \\ 08015 Barcelona, baiget@sarenet.es
}

\begin{abstract}
Resumen
Revisión de los productos y servicios puestos en marcha o participados por la revista El Profesional de la Información (EPI) con el fin de ensayar nuevas formas de difusión de información científica en el área de la biblioteconomía y la documentación. A lo largo de los años y a partir del núcleo inicial de EPI se han tejido diferentes sistemas de información interrelacionados, en especial en los últimos tiempos en los que con internet resulta tan fácil estar conectado y establecer enlaces de todo tipo. Si bien cualquier esfuerzo de difusión es positivo, se constata el poco éxito de las actuales aplicaciones del movimiento 2.0 en el campo científico.
\end{abstract}

Palabras clave: Revistas científicas. Comunicación científica. Biblioteconomía y documentación. Web 2.0. Networking. Redes científicas. Redes sociales. Comunidades profesionales.

\section{Introducción}

Entre las cuestiones clave que afectan a la comunicación científica están la publicación en acceso abierto (OA), la discutida pero insustituible revisión inter pares, los repositorios institucionales, la producción de varias versiones de los mismos artículos, el aumento general de la conciencia sobre derechos de autor, el archivo y conservación, y la existencia de herramientas de comunicación más rápidas como los blogs, sitios web, feeds RSS y podcasts. Éstas, aceleran la comunicación de la ciencia, pero circunvalan a las revistas. Los académicos pueden comunicarse por los nuevos canales y las revistas quizá deban tener un papel diferente del pasado, aunque sigue siendo indispensable que los hallazgos científicos queden registrados de una manera formal.

\section{Necesidad de experimentar}

El estado actual de incertidumbre y la falta de un camino claro para el futuro han llevado a la revista El profesional de la información (EPI) a ensayar nuevas vías de comunicación dentro de la comunidad científica y profesional de la $\mathrm{Bi}$ -

\begin{abstract}
Review of products and services launched or participated by the journal El Profesional de la Información (EPI) to test new ways of disseminating scientific information in the field of Library and Information Science. From the very beginning and throughout the years, the EPI staff has built various interconnected information systems, but especially in recent times when the internet makes it so easy to be connected and to establish links of all kinds. While any effort to spread knowledge is positive, current applications of the 2.0 movement have had limited success in the scientific field.
\end{abstract}

Keywords: Scientific journals. Scientific communication. Library and Information Science. Web 2.0. Networking. Scientific networks. Social networks. Professional communities.

blioteconomía, la Documentación y la Información a quien sirve. De hecho EPI empezó a trabajar en las redes sociales desde mucho antes de su explosión con la internet, creando en 1993 la lista de distribución de correo electrónico IweTel y en 1994 el Directorio DirIWE (hoy convertido en el Directorio EXIT). La principal característica permanente de las experiencias ha sido su componente humano, algo que, a nuestro entender erróneamente, algunos creen que le resta rigor científico a la comunicación. Nosotros creemos que una cosa no quita la otra, y constantemente nos lo demuestra el éxito de los encuentros físicos (congresos, reuniones...) y virtuales (redes sociales en la Web). Sea cual sea la profesión ejercida, el hombre es un ser social que necesita el contacto con los demás, y es en los encuentros donde se acostumbran a iniciar los nuevos proyectos colaborativos.

\subsection{El Profesional de la Información}

EPI empezó a publicarse con el nombre Information World en Español (IWE) por la editorial Learned Information, Oxford, Reino Unido, tomando como modelo otra revista de la misma editorial: Information World Review (IWR) que 
hoy sigue publicándose a cargo de Incisive Media, Londres. Durante 5 años se estuvo redactando en Barcelona y e imprimiéndose en Oxford, con un formato de magazine o newsletter de noticias, experiencias, análisis y reportajes.

En 1997 fue comprada por Swets \& Zeitlinger, empresa más conocida como agencia de suscripciones de revistas con la central en Lisse, Holanda, pero por esa época también publicaba 42 revistas científicas. A petición de S\&Z, desde 1998 EPI publica artículos revisados por pares (peer review) en doble ciego -autores y referees no se conocen entre sí-, aunque siguió conservando una importante sección de notas, experiencias y reportajes.

En 2004 S\&Z vendió sus revistas a la editorial inglesa Taylor \& Francis, y, finalmente, después de 13 años de gestión a cargo de empresas extranjeras, en 2005 EPI fue adquirida por la editorial española EPI SCP, Barcelona.

Puede decirse que desde el primer momento la Redacción estuvo obsesionada por la calidad (aunque ello ha requerido todo un proceso de aprendizaje a lo largo de los años): se revisan los textos, se comprueban los URLs, se mejora la redacción y la legibilidad, se añaden aclaraciones y definiciones, se despliegan siglas, se mejoran tablas y gráficos, se revisa la bibliografía... Por término medio, a cada artículo se dedican unas 7 horas de trabajo. Todo este esfuerzo, junto a su puntualidad y visibilidad internacional, fue reconocido en 2006 cuando empezó a ser indexada por Thomson-Reuters ISI (Web of Science) y en 2007 por Elsevier (base de datos Scopus)

\subsubsection{Construyendo comunidad: fotos de los autores}

La fotografías de los autores de los artículos y de los protagonistas de las noticias se han incluido desde el primer número de la revista como medio de networking profesional, para crear "comunidad", y aunque al principio fue algo muy criticado —considerado por algunos "poco científico-, a la larga ha resultado un éxito por lo mucho que ha ayudado a conocernos y a colaborar.

Sin embargo, publicar las fotos de los autores no es sencillo y da muchísimo trabajo. Algunas personas se niegan a fotografiarse por superstición, miedo o timidez, y una gran mayoría muestra desidia y descuido. Existe una enorme falta de "cultura" fotográfica que se traduce en la práctica por desconocer los formatos y los tamaños de ficheros más corrientes. Así, por ejemplo, es frecuente recibir en la Redacción ficheros de sólo $3 \mathrm{~KB}$ en jpg para publicar en papel, y cantidad de defectos como contraluces, desenfoques (especialmente en mujeres entradas en años que desconocen el uso del Photoshop), fotos del despacho en vez de la persona, fotos de grupo, con gafas de sol... Las fotos de mala calidad desmerecen el texto que acompañan.

Es frecuente también que los autores intenten la cansina repetición durante años y años de la misma foto, lo cual demuestra pobreza tanto por su parte como de la revista que la publica.

La sensibilidad hacia la calidad de las fotografías es un indicio del nivel cultural de las personas y de las comunidades. Existen excepciones de todo tipo, pero grosso modo podría establecerse una correlación entre la cultura fotográfica y la riqueza de los países.

\subsubsection{EPI electrónico}

Poner una revista en la Red significa crear nuevas vías de difusión, nuevos canales y, en definitiva, más enlaces y más red para la misma, por lo cual su visibilidad aumenta enormemente. EPI lleva ya 8 años online (desde 2000) y se distribuye desde MetaPress, empresa del grupo Ebsco (acceso de pago, 2000-actualidad), y desde una web propia (acceso gratuito desde 1992-actualidad, excepto embargo 2 años).

Usar un distribuidor internacional de gran calidad de servicio, y estar junto a editoriales como Taylor \& Francis o Springer, ha sido una buena inversión para la revista, pues ha ayudado a tener una imagen más profesional y creíble a la hora de relacionarse con instituciones y empresas extranjeras.

Para la gestión de los textos se usa el programa OJS instalado en el Recyt (Repositorio Español de Ciencia y Tecnología), de la Fecyt (Fundación Española para la Ciencia y la Tecnología).

\subsubsection{Modelos de financiación y Open Access (OA)}

Aunque esta comunicación se refiere a comunicación científica, hay que hablar de los modelos económicos que la hacen viable. EPI está ensayando varias de las formas existentes de vender la información, teniendo en cuenta que tiene que autofinanciarse al tratarse de una revista completamente comercial que no recibe ninguna subvención.

Además de las más usuales como suscripción anual, pago por artículo (pay per view) y pago por fascículo, también ofrece la opción "autor paga": un autor o su institución abonan una cantidad (que actualmente es sólo de 300 eu- 
ros) y liberan su artículo, que de esta manera se deja en acceso abierto tanto en MetaPress como en la web de la revista. Esto es conocido como "ruta dorada" al acceso abierto.

La revista apoya así mismo la llamada "ruta verde" al OA: permite que los autores suban sus trabajos a todas las webs y repositorios que deseen inmediatamente después que se han publicado. Por su parte, la revista deja en abierto todos los números pasado un embargo o time wall de 24 meses.

\subsection{Lista de correo-e IweTel}

Aunque importante, una revista considerada aisladamente "sólo" es un canal de comunicación, pero en la práctica hacen falta otros. A partir de la misma, su personal ha ido montando otros canales y redes adaptados a diferentes necesidades informativas: lista-e, directorio, blog, sistema de noticias, análisis, etc., creando así una compleja infraestructura alrededor.

En 1993 estableció la lista de correo-e IweTel (tomando las siglas del nombre Information World en Español y del proveedor de servicios internet Spritel, hoy Sarenet, donde se alojó hasta 1997). La lista, hoy en los servidores de RedIRIS, tiene 4.300 inscritos (mayo de 2009).

Es interesante constatar que las listas de distribución de correo electrónico se inventaron hace ya 30 años, pero pese (o gracias) a su sencillez, comodidad y mínima intrusividad (los mensajes se reciben silenciosamente y se leen cuando se dispone de tiempo) no se han visto excesivamente superadas todavía por otros sistemas. Algunos pocos blogs sí que están atrayendo alguna pequeña parte de ese bien tan preciado que es la atención y el tiempo de los bibliotecarios y documentalistas, especialmente los blogs más potentes realizados por equipos de profesionales, como el de Sedic, o también la web de votación de noticias DocuMenea. Sin embargo los blogs no son tan cómodos como las listas para mantener debates.

\subsection{Directorio de expertos EXIT}

La dirección de EPI pensó que sería interesante y útil conocernos las caras autores, lectores y demás profesionales de la información, por lo que con Josep-Manuel Rodríguez-Gairín (Kronosdoc) puso en marcha un directorio online con fotografías. El fin primario de un directorio es facilitar los datos de localización de otras personas e instituciones para poder ponerse en contacto con ellas. Pero en realidad es mucho más que esto: es un instrumento de vertebración, como la espina dorsal de una comunidad, una forma de definirla a base de enumerar cada uno de sus miembros. Establece una ordenación sistemática de personas, permite ver quién está y quién no, compararnos con los demás, emular la situación de otros...

En definitiva, un directorio es una estructura que sirve para que sus miembros se conozcan mejor. Sin directorios también se puede funcionar, pero con toda seguridad más lentamente y peor.

Y las ventajas de un directorio impreso se magnifican cuando se pone en línea, pues a la facilidad de actualización por parte de cada interesado y la búsqueda por distintos criterios se suma la comodidad de tener enlaces desde cada ficha a cualquier tipo de información externa.

EXIT (Directorio de Expertos en el Tratamiento de la Información) se creó en febrero-mayo de 2005. En mayo de 2009 cuenta con 1.600 fichas de profesionales de la biblioteconomía y la documentación, y ya empieza a ser un referente de ámbito global. La visibilidad de EXIT atrae actualmente unas 25.000 visitas mensuales y su estigmergia invita a que se suscriban espontáneamente un promedio de 5 profesionales a la semana.

\subsection{Entorno Ciencia 2.0}

En plena era 2.0 la revista se ha planteado la viabilidad de una hipotética "revista EPI 2.0".

Pero antes vayamos a la definición: A nuestro entender, una aplicación 2.0 es la que se hace de forma compartida y abierta (con contribuciones de gente que puede ser desconocida), por internet, para obtener un resultado superior.

O sea, en un producto o servicio 2.0 las aportaciones de los colaboradores externos en conjunto son más importantes que las del equipo administrador.

Evidentemente, es la gente la que hace que algo sea 2.0. Si se instala una plataforma "2.0" en la Red y no la usa nadie, no podrá ser 2.0.

Dada la moda actual, con tantos bloggers haciendo resonar sus actividades por la Red, quedaría bien y moderno poder decir que EPI es 2.0. Y además, si fuera verdad, sería muy descansado para la Redacción, que vería cómo se van llenando de materiales las páginas del siguiente número... Pero no es así. Creemos que una revista científica clásica difícilmente puede ser 2.0, pues se trata de un producto sofisticado, con un rígido, estricto y a la vez ágil proceso de elaboración. Algún otro producto con características especiales, como por ejemplo Wikipedia, es viable por tratarse de miles de pequeños artículos, básicamente estáticos e independien- 
tes, de saber ya registrado previamente. Pero es muy difícil trasladar ese modelo de trabajo a una revista debido a su difícil y dinámica elaboración. Lo más a que puede aspirar una revista científica puntera que publica artículos originales es a establecer un blog o una lista de discusión sobre los artículos publicados, o temas relacionados.

Pero si EPI no es 2.0, el equipo de la revista sí intenta trabajar en entornos que lo son, o que por lo menos tienen algunas de las características que hemos citado: compartir, estar abiertos a contribuciones por internet, para obtener un resultado superior. Algunas de las actividades realizadas se han basado en el uso de redes y plataformas ya existentes, como un grupo de discusión y noticias en Facebook, y un canal en Twitter.

Y en la web se ha creado el blog Zona de notas donde se recopilan actividades en las que participa la Redacción.

Tanto por nuestra experiencia como por la que vemos en otras revistas, estos sistemas de comunicación no han tenido éxito, al menos hasta ahora. Las posibles razones son:

- Existen ya demasiados lugares donde poder intercambiar opiniones. Ninguna de las nuevas plataformas aporta ventajas sobre las sencillas y veteranas listas de correo-e.

- Poco interés en tratar temas generales, que son los que podrían ser comunes a todo un grupo.

- Los investigadores sólo tienen necesidad de discutir con colegas próximos a su especialidad, capaces de entender sus problemas y de aportarles ideas y opiniones útiles para sus proyectos.

\subsection{DocuMenea}

Se trata de una web de promoción de noticias de biblioteconomía, documentación, informática, periodismo, etc., al estilo de Digg y Menéame.

Su promotor fue un equipo de 9 profesionales relacionados con $\mathrm{EPI}$, a los que se han sumado otros voluntarios. Cualquier persona puede introducir noticias en el sistema, y todos pueden votar ("menear") las noticias más interesantes, que de esta manera adquieren más visibilidad al pasar a la página principal.

A pesar de ofrecer una excelente y exhaustiva revisión de todas las noticias que pueden interesar en nuestro sector, incluso traducidas al español, es un servicio relativamente poco usado por la gran mayoría de colegas, lo que una vez más viene a demostrar la poca aceptación de los productos web 2.0 en campos especializados.

\subsection{IraLIS, "normalización" de nombres de autor}

Cuando EPI empezó a estar indizado en las bases de datos internacionales observamos que sistemáticamente todos los autores españoles aparecian por su segundo apellido. El hecho nos es conocido desde hace muchos años, pues los anglosajones siempre nos han indizado así cuando firmamos con 2 apellidos, pero el verlo tan "de cerca" con los artículos de la revista nos hizo llegar a una clara conclusión: los hispanos no podemos continuar firmando diferente del resto del mundo, tenemos que adaptarnos a los demás.

Por ello creamos la plataforma IraLIS () y obligamos a todos los autores de la revista a firmar con un formato "iralizado": un solo nombre y un solo apellido. Si se quieren usar dos nombres o dos apellidos, deben juntarlos completamente 0 con un guión, a pesar de que el "apellido" resultante sea uno compuesto artificialmente.

\subsection{E-LIS}

E-prints in Library; Information Science es un repositorio de documentos sobre biblioteconomía, documentación y ciencias de la información creado en 2003 y alojado en el Consorzio Interuniversitario Lombardo per Elaborazione Automatica (Cilea). Desde sus inicios E-LIS ha estado subvencionado por el Ministerio de Cultura de España.

Viendo la importancia del movimiento de acceso abierto, y en particular el interés de E-LIS como factor aglutinador de la comunidad de ByD, la dirección de EPI se involucró en el proyecto, siendo ahora editora de las aportaciones españolas al repositorio. Los bibliotecariosdocumentalistas españoles son los que más documentos introducen en el sistema.

Los documentos depositados en E-LIS no solamente gozan de mucha más visibilidad —el servidor sirve 175.000 trabajos mensuales y cada artículo en texto completo tiene una media de 18,5 descargas mensuales-, sino que tienen garantizada su conservación a largo plazo. Por ello desde EPI se contribuye a difundir la existencia del repositorio y a invitar a los autores a que depositen sus artículos (vía verde al OA).

\section{Conclusiones}

Desde sus mismos inicios, la dirección de la revista EPI siempre tuvo en mente la comunidad 
de profesionales de la documentación, no como simples lectores sino como personas, con nombres y apellidos (y si era posible con fotografías) para incentivar la cooperación y activar su desarrollo. La puesta en marcha de EPI en 1992 primeramente como boletín favorecía la posibilidad de tratar los temas de una forma más personal y desenfadada. Posteriormente, en 1998, al solicitar la editorial su transformación en revista "científica", la Redacción tuvo que cuidar más los aspectos formales.

Sin embargo, siguiendo con la idea de que las relaciones humanas son el motor que mueve la mayoría de proyectos, a lo largo de los años el equipo de personas de EPI ha ensayado diversas estructuras para entretejer redes de comunicación, colaboración e intercambio, y participando en la organización y funcionamiento de sociedad profesionales.

Con la llegada de internet se han multiplicado las vías de comunicación, y a pesar de que el número de profesionales en España ha crecido enormemente con la implantación de los estudios universitarios de Biblioteconomía y Documentación, quizá sea ahora más fácil que nunca que la gente se conozca.

La segunda oleada de internet, la llamada Web 2.0 o participativa, creó a partir de 2004 expectativas para establecer nuevos sistemas de difusión de la ciencia. Sin embargo los sistemas actuales, que tan buena acogida tienen para el ocio y las relaciones de amistad, no parecen tener mucho interés para el trabajo de los investigadores, a juzgar por lo poco que los usan. Existen muchos y diversos ejemplos de esa indiferencia que van desde Nature Preceedings, de la revista Nature, al grupo de Facebook de la revista EPI.

Si bien Facebook no juega un papel importante en la difusión de la ciencia ni para el trabajo en general, sí lo hace en cuanto a ayudar a establecer relaciones de conocimiento y amistad entre colegas, cosa que también resulta positiva.

Por último, hay que decir que la revista ha tenido que luchar para conseguir ser viable económicamente como revista $100 \%$ comercial. Creemos que todos los esfuerzos de creación de comunidad y de relaciones personales han ayudado mucho a dicho fin; de no ser por ellos probablemente EPI ya no existiría.

\section{Apéndice 1. Direcciones URL de EPI y herramientas asociados}

Revista El profesional de la información

- Web propia http://www.elprofesionaldelainformacion.com
- Web de distribución http://elprofesionaldelainformacion.metapress.com

- Web de producción http://fecyt.recyt.es/EPI

- Blog EPI (Zona de notas) http://www.elprofesionaldelainformacion.com/notas/

- Página EPI en Facebook http://www.facebook.com/group.php?gid=36050316757

- Canal EPI en Twitter: http://twitter.com/Revista_EPI

Lista de distribución de correo-e IweTel http://www.rediris.es/list/info/iwetel.html

Directorio EXIT. http://www.directorioexit.info

Promotor de noticias Documenta. http://www.documenea.com

Normalizador de nombres de autor. http://www.iralis.org

Repositorio E-LIS http://eprints.rclis.org/

\section{Referencias}

Abadal, Ernest; Rius Alcaraz, Lluís (2008). Revistas científicas de las universidades españolas: acciones básicas para aumentar su difusión e impacto. // Revista Española de Documentación Científica. $31: 2$ (2008) 242262. http://eprints.rclis.org/13422

Almeida, Robson (2008). Disseminação de conteúdos na Web: a tecnologia RSS como proposta para a comunicação científica. Ms. thesis, Universidade de Brasília. http://eprints.rclis.org/13971

Alonso-Arévalo, Julio (2004) Comunicación científica y edición alternativa: Visibilidad y fuentes de información en ByD. http://eprints.rclis.org/4976

Alonso-Arévalo, Julio; Subirats Coll, Imma; Martínez-Conde, María-Luisa (2008). Informe APEI sobre acceso abierto. // Informes APEI (Asociación Profesional de Especialistas en Información, Spain). http://eprints.rclis.org/15107

Baiget, Tomàs; Rodríguez-Gairín, Josep-Manuel; Peset, Fernanda; Subirats, Imma; Ferrer-Sapena, Antonia (2007). Normalización de la información: la aportación de IraLIS. // El profesional de la información. 16:6 (nov.dic. 2007) 636-643.

Drake, Miriam A. (2007). "cholarly communication in turmoil. // Information Today. 24:2 (feb. 2007) 1, 18-19. http://www.infotoday.com/it/feb07/Drake.shtml

Fernández-Morales, Isabel (2003). El impacto de los archivos de e-prints en la comunicación científica entre los investigadores españoles: aceptación y uso. http://eprints.rclis.org/10045

Gómez, Nancy D.; Arias, Olga M. (2002). El cambio de paradigma en la comunicación científica. // Información, cultura y sociedad. 6. http://eprints.rclis.org/3649

Gómez-Dueñas, Laureano-Felipe (2006). La Iniciativa de Archivos Abiertos (OAI): Un nuevo paradigma en la comunicación científica y el intercambio de información. // Revista Códice. 2:1 (2006) 21-48. http://eprints. rclis.org/9832

Orduña-Malea, Enrique; Rodríguez-Gairín, Josep-Manuel; Baiget, Tomàs (2007). Directorio de expertos en el tratamiento de la información (EXIT). Análisis de uso. // El profesional de la información. 16:5 (sept.-oct. 2007) 497-509.

Rodríguez-López, Joaquín (2005). Ciencia y comunicación científica: edición digital y otros fundamentos del libre acceso al conocimiento. // El profesional de la informa- 
ción. 14:4 (2005). http://www.elprofesionaldelainforma cion.com/contenidos/2005/noviembre/2.pdf

Sosa, Miguel; Soria, Verónica (2004). La comunicación científica a través de los e-print. // Foro Internacional Sobre Tecnologías de Información, Interfaces 2004, Colima (México), 22-26 nov. http://eprints.rclis.org/6768

Torres-Salinas, Daniel; Guallar, Javier (2009). Evaluación de DocuMenea, sistema de promoción social de noticias de biblioteconomía y documentación. // El profesional de la información. 18: 2 (marzo-abril 2009) 171-179.

Torres-Salinas, Daniel; Cabezas-Clavijo, Álvaro (2008). Los blogs como nuevo de comunicación científica. // III Encuentro Ibérico de Docentes e Investigadores en Información y Documentación, Salamanca (Spain), 5-7 mayo. http://eprints.rclis.org/13385 